\title{
Fluid Updates: Beyond Strong vs. Weak Updates ${ }^{\star}$
}

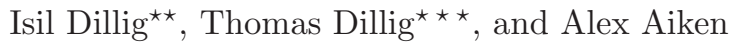 \\ Department of Computer Science, Stanford University \\ \{isil,tdillig, aiken\}@cs.stanford.edu
}

\begin{abstract}
We describe a symbolic heap abstraction that unifies reasoning about arrays, pointers, and scalars, and we define a fluid update operation on this symbolic heap that relaxes the dichotomy between strong and weak updates. Our technique is fully automatic, does not suffer from the kind of state-space explosion problem partition-based approaches are prone to, and can naturally express properties that hold for non-contiguous array elements. We demonstrate the effectiveness of this technique by evaluating it on challenging array benchmarks and by automatically verifying buffer accesses and dereferences in five Unix Coreutils applications with no annotations or false alarms.
\end{abstract}

\section{Introduction}

In existing work on pointer and shape analysis, there is a fundamental distinction between two kinds of updates to memory locations: weak updates and strong updates [1 4]. A strong update overwrites the old content of an abstract memory location $l$ with a new value, whereas a weak update adds new values to the existing set of values associated with $l$. Whenever safe, it is preferable to apply strong updates to achieve better precision.

Applying strong updates to abstract location $l$ requires that $l$ correspond to exactly one concrete location. This requirement poses a difficulty for applying strong updates to (potentially) unbounded data structures, such as arrays and lists, since the number of elements may be unknown at analysis time. Many techniques combine all elements of an unbounded data structure into a single summary location and only allow weak updates [2, [5, 6]. More sophisticated techniques, such as analyses based on 3-valued logic [3], first isolate individual elements of an unbounded data structure via a focus operation to apply a strong update, and the isolated element is folded back into the summary location via a dual blur operation to avoid creating an unbounded number of locations. While such an approach allows precise reasoning about unbounded data structures, finding the right focus and blur strategies can be challenging and hard to automate [3].

\footnotetext{
* This work was supported by grants from NSF (CNS-050955 and CCF-0430378) with additional support from DARPA.

** Supported by a Stanford Graduate Fellowship.

$\star \star \star$ Supported by a Siebel Fellowship.
} 
In this paper, we propose a way of relaxing the dichotomy between applying weak vs. strong updates to a particular kind of unbounded data structure, arrays, by introducing fluid updates. Fluid updates can always be safely applied regardless of whether a given abstract memory location represents a single concrete location or an array. Three key ideas underpin fluid updates:

1. Arrays are modeled as abstract locations qualified by index variables; constraints on index variables specify which concrete elements are referred to by a points-to edge.

2. In general, we may not know the exact subset of concrete elements updated by a statement. To deal with this uncertainty, each points-to edge is qualified by a pair of constraints $\left\langle\phi_{N C}, \phi_{S C}\right\rangle$, called bracketing constraints, over- and underapproximating the subset of concrete elements selected by this edge.

3. To apply a fluid update, we compute a bracketing constraint $\left\langle\phi_{N C}, \phi_{S C}\right\rangle$ representing over- and underapproximations for the set of concrete elements updated by a statement. A fluid update preserves all existing points-to edges under the negation of the update condition, i.e., $\neg\left\langle\phi_{N C}, \phi_{S C}\right\rangle=\left\langle\neg \phi_{S C}, \neg \phi_{N C}\right\rangle$, while applying the update under $\left\langle\phi_{N C}, \phi_{S C}\right\rangle$.

An important property of bracketing constraints is that the intersection of a bracketing constraint $B$ and its negation $\neg B$ is not necessarily empty (see Section 2.1). For array elements in the intersection, both the new value is added and the old values are retained - i.e., a weak update is performed. Because fluid updates rely on negation, having both over- and underapproximations (or equivalently, necessary and sufficient conditions) is crucial for the correctness of our approach.

If the concrete elements updated by a statement $s$ are known exactly, i.e., $\phi_{N C}$ and $\phi_{S C}$ are the same, the fluid update represents a strong update to some set of elements in the array. On the other hand, if nothing is known about the update condition, i.e., $\left\langle\phi_{N C}, \phi_{S C}\right\rangle=\langle$ true, false $\rangle$, the fluid update is equivalent to a weak update to all elements in the array. Otherwise, if only partial information is available about the concrete elements modified by $s$, the fluid update encodes this partial information soundly and precisely. Consider the following example:

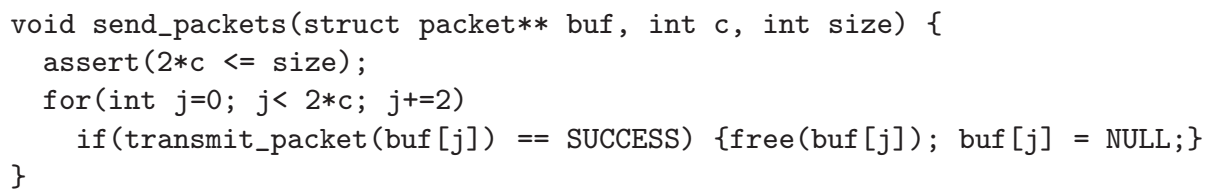

The function send_packets takes an array buf of packet*'s, an integer c representing the number of high-priority packets to be sent, and an integer size, denoting the number of elements in buf. All even indices in buf correspond to high-priority packets whereas all odd indices are low-priority 11 This function submits one high-priority packet at a time; if the transfer is successful (which may

${ }^{1}$ The distinction between even and odd-numbered elements in a network buffer arises in many real network applications, for example in packet scheduling [7] and $\mathrm{p} 2 \mathrm{p}$ video streaming [8] 
depend on network traffic), it sets the corresponding element in buf to NULL to indicate the packet has been processed.

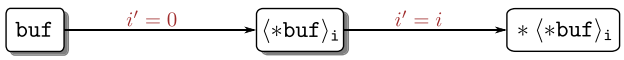

The figure above shows the symbolic heap abstraction at the entry of send_packets. Here, nodes represent abstract locations named by access paths [9], and edges denote points-to relations. Because either the source or target of an edge may be a set, we write constraints on edges to indicate which elements of the source point to which elements of the target. In the figure, the dereference of buf is an array, hence, it is qualified by an index variable $i$; the location named $\langle * \text { buf }\rangle_{\text {i }}$ represents all elements of array $*$ buf. By convention, primed index variables on an edge qualify the edge's target, and unprimed index variables qualify the source. If the overand underapproximations on an edge are the same, we write a single constraint instead of a pair. In this graph, the edge from buf to $\langle * \text { buf }\rangle_{i}$ is qualified by $i^{\prime}=0$ because buf points to the first element of array $\langle * \text { buf }\rangle_{i}$. The constraint $i=i^{\prime}$ on the edge from $\langle * \text { buf }\rangle_{i}$ to $*\langle * \text { buf }\rangle_{i}$ indicates that the $i$ 'th element of array $*$ buf points to some corresponding target called $*\langle * \text { buf }\rangle_{i}$.

The concrete elements modified by the statement buf $[j]=$ NULL cannot be specified exactly at analysis time since the success of transmit_packet depends on an environment choice (i.e., network state). The loop may, but does not have to, set all even elements between 0 and $2 c$ to NULL. Hence, the best overapproximation of the indices of $*$ buf modified by this statement is $0 \leq i<$ $2 c \wedge i \% 2=0$. On the other hand, the best underapproximation of the set of indices updated in the loop is the empty set (indicated by the constraint false) since no element is guaranteed to be updated by the statement buf $[j]=$ NULL.

Figure 1/shows the symbolic heap abstraction at the end of send_packets. Since the set of concrete elements that may be updated by buf $[j]=$ NULL is given by $\langle 0 \leq i<2 c \wedge i \% 2=0$, false $\rangle$, the fluid update adds an edge from $\langle * \text { buf }\rangle_{i}$ to $*$ NULL under this bracketing constraint. The existing edge from $\langle * \mathrm{buf}\rangle_{\mathrm{i}}$ to $*\langle * \mathrm{buf}\rangle_{\mathrm{i}}$ is preserved under $\neg\langle 0 \leq i<2 c \wedge i \% 2=0$, false $\rangle$. Now, the complement (negation) of an overapproximation is an underapproximation of the complement; similarly the complement of an underapproximation is an overapproximation of the complement. Thus, assuming $i \geq 0$, this is equivalent to $\langle$ true, $i \geq 2 c \vee i \% 2 \neq 0\rangle$. Since the initial constraint on the edge stipulates $i=i^{\prime}$, the edge constraint after the fluid update becomes $\left\langle i=i^{\prime},(i \geq 2 c \vee i \% 2 \neq 0) \wedge i=i^{\prime}\right\rangle$. The new edge condition correctly and precisely states that any element of $*$ buf may still point to its original target when the function exits, but only those elements whose index satisfies the constraint $i \geq 2 c$ or $i \% 2 \neq 0$ must point to their original target. As this example illustrates, fluid updates have the following characteristics:

- Fluid updates do not require concretizing individual elements of an array to perform updates, making operations such as focus and blur unnecessary.

- Fluid updates never construct explicit partitions of an array, making this approach less vulnerable to the kind of state space explosion problem that partition-based approaches, such as [3], are prone to. 


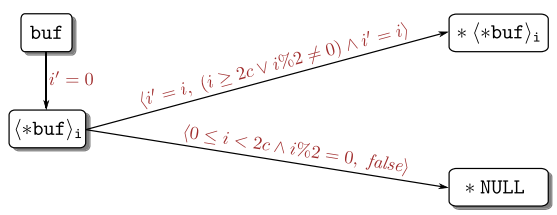

Fig. 1. The points-to graph at the end of function send_packets

- Fluid updates preserve partial information despite imprecision and uncertainty. In the above example, although the result of transmit_packet is unknown, the analysis can still determine that no odd packet is set to NULL.

- Fluid updates separate the problem of determining which concrete elements are updated from how the update is performed. Fluid updates are oblivious to the precision of the over- and underapproximations, and retain the best possible information with respect to these approximations. In the above example, a less precise overapproximation, such as $0 \leq i<2 c$, would not affect the way updates are performed.

This paper is organized as follows: Section 2 defines a simple language and introduces basic concepts. Section 3 formalizes the symbolic heap abstraction, Section 4 presents the basic pointer and value analysis based on fluid updates, and Section 5 discusses treatment of loops. Section 6 discusses a prototype implementation, Section 7 presents our experimental results, and Section 8 surveys related work. To summarize, this paper makes the following key contributions:

- We introduce fluid updates as a viable alternative to the dichotomy between weak vs. strong updates, and we describe an expressive memory analysis based on symbolic heap abstraction that unifies reasoning about arrays, pointers, and scalars. (We do not, however, address recursive pointer-based data structures in this paper.)

- We propose bracketing constraints to allow a sound negation operation when performing updates in the presence of imprecision and uncertainty.

- We demonstrate our technique is precise and efficient for reasoning about values and points-to targets of array elements. Furthermore, our technique is fully automatic, requiring no annotations or user-provided predicates.

- We show the effectiveness of our approach by verifying the safety of buffer accesses and dereferences fully automatically in five Unix Coreutils applications that manipulate arrays and pointers in intricate ways.

\section{Language and Preliminaries}

We first define a small imperative language in which we formalize our technique:

$$
\begin{aligned}
\text { Program } P:= & F^{+} \\
\text {Function } F:= & \text { define } f\left(v_{1}, \ldots, v_{n}\right)=S \\
\text { Statement } S:= & S_{1} ; S_{2}\left|v_{1}=v_{2}\right| v_{1}=c\left|v_{1}=\operatorname{alloc}\left(v_{2}\right)\right| v_{1}=v_{2}\left[v_{3}\right] \mid v_{2}\left[v_{3}\right]=v_{1} \\
& \left|v_{1}=v_{2} \oplus v_{3}\right| v_{1}=v_{2} \text { intop } v_{3} \mid v_{1}=v_{2} \text { predop } v_{3} \mid \\
& \text { if } v \neq 0 \text { then } S_{1} \text { else } S_{2} \mid \text { while } v \neq 0 \text { do } S \text { end }
\end{aligned}
$$


In this grammar, $v$ is a variable, and $c$ is an integer constant. Types are defined by the grammar $\tau:=$ int $\mid \operatorname{pointer}(\operatorname{array}(\tau))$. Load $\left(v_{1}=v_{2}\left[v_{3}\right]\right)$ and store $\left(v_{2}\left[v_{3}\right]=v_{1}\right)$ statements are defined on pointers $v_{2}$ and integers $v_{3}$, and we assume programs are well-typed. $v[i]$ first dereferences $v$ and then selects the $i$ 'th element of the array pointed to by $v$. Pointer arithmetic $v_{1}=v_{2} \oplus v_{3}$ makes $v_{1}$ point to offset $v_{3}$ in the array pointed to by $v_{2}$. Integer operations (intop) include,+- , and $\times$. Predicate operators (predop) are $=, \neq$ and $<$, and predicates evaluate to 0 (false) or 1 (true). The alloc $\left(v_{2}\right)$ statement allocates an array with $v_{2}$ elements.

An operational semantics for this language is given in the extended version of this paper [30]. In the concrete semantics, a concrete location $l_{c}$ is a pair $(s, i)$ where $s$ is a start address for a block of memory and $i$ is an offset from $s$. An environment $E$ maps program variables to concrete locations, and a store $S$ maps locations to other locations or integer values. Due to space limitations, we omit function calls from our formal discussion; Section 6 discusses how we treat function calls in the implementation.

\subsection{Constraint Language}

The constraints used in the analysis are defined by:

$\begin{array}{ll}\text { Term } T & :=c|v| T_{1} \text { intop } T_{2}\left|\operatorname{select}\left(T_{1}, T_{2}\right)\right| \operatorname{deref}(T) \\ \text { Literal } L & :=\text { true } \mid \text { false } \mid T_{1} \text { predop } T_{2} \mid T \% c=0 \\ \text { Atom } A & :=L|\neg A| A_{1} \wedge A_{2} \mid A_{1} \vee A_{2} \\ \text { Constraint } C & :=\left\langle A_{N C}, A_{S C}\right\rangle\end{array}$

Terms are constants, variables, arithmetic terms, and the uninterpreted function terms $\operatorname{select}\left(T_{1}, T_{2}\right)$, and $\operatorname{deref}(T)$. Terms are used to represent scalars, pointers, and arrays; the uninterpreted function term $\operatorname{select}\left(T_{1}, T_{2}\right)$ represents the result of selecting element at index $T_{2}$ of array $T_{1}$, and the term $\operatorname{deref}(T)$ represents the result of dereferencing $T$.

Literals are true, false, comparisons $(=, \neq,<)$ between two terms, and divisibility checks on terms. Atomic constraints $A$ are arbitrary boolean combinations of literals. Satisfiability and validity of atomic constraints are decided over the combined theory of uninterpreted functions and linear integer arithmetic extended with divisibility (mod) predicates. Bracketing constraints $C$ are pairs of atomic constraints of the form $\left\langle A_{N C}, A_{S C}\right\rangle$ representing necessary and sufficient conditions for some fact. A bracketing constraint is well-formed if and only if $A_{S C} \Rightarrow A_{N C}$. We write $\lceil\phi\rceil$ to denote the necessary condition of a bracketing constraint $\phi$ and $\lfloor\phi\rfloor$ to denote the sufficient condition of $\phi$.

Example 1. Consider an edge from location $\langle * \mathrm{a}\rangle_{\mathrm{i}}$ to $*$ NULL qualified by $\langle 0 \leq i<$ size, $0 \leq i<$ size $\rangle$.This constraint expresses that all elements of the array with indices between 0 and size are NULL. Since it is sufficient that $i$ is between 0 and size for $\langle * \mathrm{a}\rangle_{\text {i }}$ to point to $*$ NULL, it follows that all elements in this range are NULL. On the other hand, if the constraint on the edge is $\langle 0 \leq i<$ size, false $\rangle$, any element in the array may be NULL, but no element must be NULL. 
Boolean operators $\neg, \wedge$, and $\vee$ on bracketing constraints are defined as:

$$
\begin{aligned}
\neg\left\langle A_{N C}, A_{S C}\right\rangle & =\left\langle\neg A_{S C}, \neg A_{N C}\right\rangle \\
\left\langle A_{N C 1}, A_{S C 1}\right\rangle \star\left\langle A_{N C 2}, A_{S C 2}\right\rangle & =\left\langle A_{N C 1} \star A_{N C 2}, A_{S C 1} \star A_{S C 2}\right\rangle \quad(\star \in\{\wedge, \vee\})
\end{aligned}
$$

Since the negation of the overapproximation for some set $S$ is an underapproximation for the complement of $S$, necessary and sufficient conditions are swapped under negation. The following lemma is easy to show:

Lemma 1. Bracketing constraints preserve the well-formedness property $A_{\mathrm{SC}} \Rightarrow$ $A_{\mathrm{NC}}$ under boolean operations.

Satisfiability and validity are defined in the following natural way:

$$
S A T\left(\left\langle A_{N C}, A_{S C}\right\rangle\right) \equiv \operatorname{SAT}\left(A_{N C}\right) \quad \operatorname{VALID}\left(\left\langle A_{N C}, A_{S C}\right\rangle\right) \equiv \operatorname{VALID}\left(A_{S C}\right)
$$

Lemma 2. Bracketing constraints do not obey the law of the excluded middle and non-contradiction, but they satisfy the following weaker properties:

$$
\operatorname{VALID}\left(\left\lceil\left\langle A_{\mathrm{NC}}, A_{\mathrm{SC}}\right\rangle \vee \neg\left\langle A_{\mathrm{NC}}, A_{\mathrm{SC}}\right\rangle\right\rceil\right) \quad \operatorname{UNSAT}\left(\left\lfloor\left\langle A_{\mathrm{NC}}, A_{\mathrm{SC}}\right\rangle \wedge \neg\left\langle A_{\mathrm{NC}}, A_{\mathrm{SC}}\right\rangle\right\rfloor\right)
$$

Proof. $\left\lceil\left\langle A_{N C}, A_{S C}\right\rangle \vee \neg\left\langle A_{N C}, A_{S C}\right\rangle\right\rceil$ is $\left(A_{N C} \vee \neg A_{S C}\right) \Leftrightarrow\left(A_{S C} \Rightarrow A_{N C}\right) \Leftrightarrow$ true, where the last equivalence follows from well-formedness. Similarly, $\left\lfloor\left\langle A_{N C}, A_{S C}\right\rangle \wedge \neg\left\langle A_{N C}, A_{S C}\right\rangle\right\rfloor$ is $\left(A_{S C} \wedge \neg A_{N C}\right) \Leftrightarrow$ false, where the last step follows from the well-formedness property.

\section{Symbolic Heap Abstraction}

Abstract locations are named by access paths [9] and defined by the grammar:

$$
\text { Access Path } \pi:=\mathfrak{L}_{v} \mid \text { alloc }_{i d}\left|\langle\pi\rangle_{i}\right| * \pi|c| \pi_{1} \text { intop } \pi_{2} \mid \top
$$

Here, $\mathfrak{L}_{v}$ denotes the abstract location corresponding to variable $v$, and alloc $c_{i d}$ denotes locations allocated at program point $i d$. Any array location is represented by an access path $\langle\pi\rangle_{i}$, where $\pi$ represents the array and $i$ is an index variable ranging over the indices of $\pi$ (similar to [22]). The location $* \pi$ represents the dereference of $\pi$. The access path $c$ denotes constants, $\pi_{1}$ intop $\pi_{2}$ represents the result of performing intop on $\pi_{1}$ and $\pi_{2}$, and $\top$ denotes any possible value.

A memory access path, denoted $\pi_{m e m}$, is any access path that does not involve $c, \pi_{1}$ intop $\pi_{2}$, and $T$. We differentiate memory access paths because only locations that are identified by memory access paths may be written to; other kinds of access paths are only used for encoding values of scalars.

Given a concrete store $S$ and an environment $E$ mapping program variables to concrete locations, a function $\gamma$ maps abstract memory locations to a set of concrete locations $\left(s_{1}, i_{1}\right) \ldots\left(s_{k}, i_{k}\right)$ :

$$
\begin{aligned}
& \gamma\left(E, S, \mathfrak{L}_{v}\right)=\{E(v)\} \\
& \gamma(E, S, \text { alloc } i d)=\{(l, 0) \mid l \text { is the result of allocation at program point id }\} \\
& \left.\gamma\left(E, S,\langle\pi\rangle_{i}\right)=\left\{\left(l, \text { index }_{j}\right) \mid(l, \text { index }) \in S \wedge(l, 0) \in \gamma(E, S, \pi)\right)\right\} \\
& \gamma(E, S, * \pi)=\bigcup_{l_{i} \in \gamma(E, S, \pi)} S\left(l_{i}\right)
\end{aligned}
$$


Since we will concretize abstract memory locations under a certain assumption about their index variables, we define another function $\gamma_{c}$, similar to $\gamma$ but qualified by constraint $\phi$. The only interesting modification is for $\langle\pi\rangle_{i}$ :

$\gamma_{c}\left(E, S,\langle\pi\rangle_{i}, \phi\right)=\left\{\left(l\right.\right.$, index $\left._{j}\right) \mid\left(l\right.$, index $\left._{j}\right) \in S \wedge(l, 0) \in \gamma_{c}(E, S, \pi, \phi) \wedge \operatorname{SAT}\left(\phi\left[\right.\right.$ index $\left.\left.\left._{j} / i\right]\right)\right\}$

As is standard in points-to graphs, we enforce that for any two memory access paths, either $\pi_{m e m}=\pi_{m e m}^{\prime}$ or $\gamma\left(E, S, \pi_{m e m}\right) \cap \gamma\left(E, S, \pi_{m e m}^{\prime}\right)=\emptyset$.

A symbolic heap abstraction is a directed graph where nodes denote abstract locations identified by access paths and edges qualified by bracketing constraints denote points-to relations. Since we want to uniformly encode points-to and value information, we extend the notion of points-to relations to scalars. For example, if an integer a has value 3 , the symbolic heap abstraction contains a "points-to" edge from a's location to some location named * 3 , thereby encoding that the value of $\mathrm{a}$ is 3 . Hence, the symbolic heap encodes the value of each scalar.

Formally, a symbolic heap abstraction is defined by

$$
\Gamma: \pi_{m e m} \rightarrow 2^{(\pi, \phi)}
$$

mapping a source location to a set of (target location, constraint) pairs. The edge constraint $\phi$ may constrain program variables to encode the condition under which this points-to relation holds. More interestingly, $\phi$ may also qualify the source and the target location's index variables, thereby specifying which elements of the source may (and must) point to which elements of the target.

The combination of indexed locations and edge constraints parametric over these index variables makes the symbolic heap abstraction both very expressive but also non-trivial to interpret. In particular, if the source location is an array, we might want to determine the points-to targets of a specific element (or some of the elements) in this array. However, the symbolic heap abstraction does not directly provide this information since edge constraints are parametric over the source and the target's index variables. Consider the following points-to relation:

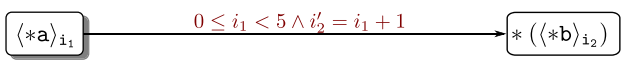

Suppose we want to know which location(s) the fourth element of array $\langle * a\rangle_{i_{1}}$ points to. Intuitively, we can determine the target of the fourth element of $\langle * a\rangle_{i_{1}}$ by substituting the index variable $i_{1}$ by value 3 in the edge constraint $0 \leq i_{1}<$ $5 \wedge i_{2}^{\prime}=i_{1}+1$. This would yield $i_{2}^{\prime}=4$, indicating that the fourth element of $\langle * a\rangle_{i}$ points to the target of the fifth element of $\langle * b\rangle_{i_{2}}$.

While a simple substitution allows us to determine the target of a specific array element as in the above example, in general, we need to determine the targets of those array elements whose indices satisfy a certain constraint. Since this constraint may not limit the index variable to a single value, determining points-to targets from an indexed symbolic heap abstraction requires existential quantifier elimination in general. In the above example, we can determine the possible targets of elements of $\langle * a\rangle_{i_{1}}$ whose indices are in the range $[0,3]$ (i.e., satisfy the constraint $0 \leq i_{1} \leq 3$ ) by eliminating $i_{1}$ from the following formula:

$$
\exists i_{1} \cdot\left(0 \leq i_{1} \leq 3 \wedge\left(0 \leq i_{1}<5 \wedge i_{2}^{\prime}=i_{1}+1\right)\right)
$$


This yields $1 \leq i_{2}^{\prime} \leq 4$, indicating that the target's index must lie in the range $[1,4]$. To formalize this intuition, we define an operation $\phi_{1} \downarrow_{I} \phi_{2}$, which yields the result of restricting constraint $\phi_{1}$ to only those values of the index variables $I$ that are consistent with $\phi_{2}$.

Definition 1. $\left(\phi_{1} \downarrow_{I} \phi_{2}\right)$ Let $\phi_{1}$ be a constraint qualifying a points-to edge and let $\phi_{2}$ be a constraint restricting the values of index variables $I$. Then,

$$
\phi_{1} \downarrow_{I} \phi_{2} \equiv \operatorname{Eliminate}\left(\exists I . \phi_{1} \wedge \phi_{2}\right)
$$

where the function Eliminate performs existential quantifier elimination.

The quantifier elimination performed in this definition is exact because index variables qualifying the source or the target never appear in uninterpreted functions in a valid symbolic heap abstraction; thus the elimination can be performed using [10].

\section{Pointer and Value Analysis Using Fluid Updates}

In this section, we give deductive rules describing the basic pointer and value analysis using fluid updates. An invariant mapping $\Sigma:$ Var $\rightarrow \pi_{\text {mem }}$ maps program variables to abstract locations, and the environment $\Gamma$ defining the symbolic heap abstraction maps memory access paths to a set of (access path, constraint) pairs. Judgments $\Sigma \vdash$ a $: \mathfrak{L}_{a}$ indicate that variable a has abstract location $\mathfrak{L}_{a}$, and judgments $\Gamma \vdash_{j} \pi_{s}:\left\langle\pi_{t_{j}, \phi_{j}}\right\rangle$ state that $\left\langle\pi_{t_{j}}, \phi_{j}\right\rangle \in \Gamma\left(\pi_{s}\right)$. Note that there may be many $\left\langle\pi_{t_{j}}, \phi_{j}\right\rangle$ pairs in $\Gamma\left(\pi_{s}\right)$, and this form of judgment is used in the rules to refer to each of them without needing to use sets.

We first explain some notation used in Figure 2. The function $U(\phi)$ replaces the primed index variables in constraint $\phi$ with their unprimed counterparts, e.g., $U\left(i_{1}^{\prime}=2\right)$ is $\left(i_{1}=2\right)$; this is necessary when traversing the points-to graph because the target location of an incoming edge becomes the source of the outgoing edge from this location. We use the notation $\Gamma \wedge \phi$ as shorthand for:

$$
\Gamma^{\prime}(\pi)=\left\{\left\langle\pi_{l}, \phi_{l} \wedge \phi\right\rangle \mid\left\langle\pi_{l}, \phi_{l}\right\rangle \in \Gamma(\pi)\right\}
$$

A union operation $\Gamma=\Gamma^{\prime} \cup \Gamma^{\prime \prime}$ on symbolic heap abstractions is defined as:

$$
\left\langle\pi^{\prime}, \phi^{\prime} \vee \phi^{\prime \prime}\right\rangle \in \Gamma(\pi) \Leftrightarrow\left\langle\pi^{\prime}, \phi^{\prime}\right\rangle \in \Gamma^{\prime}(\pi) \wedge\left\langle\pi^{\prime}, \phi^{\prime \prime}\right\rangle \in \Gamma^{\prime \prime}(\pi) .
$$

We write $\mathfrak{I}(\pi)$ to denote the set of all index variables used in $\pi$, and we say " $i$ is index of $\pi$ " if $i$ is the outermost index variable in $\pi$.

The basic rules of the pointer and value analysis using fluid updates are presented in Figure 2. We focus mainly on the inference rules involving arrays, since these rules either directly perform fluid updates (Array Store) or rely on the constraint and index-based representation that is key for fluid updates.

We start by explaining the Array Load rule. In this inference rule, each $\pi_{2_{j}}$ represents one possible points-to target of $v_{2}$ under constraint $\phi_{2_{j}}$. Because $\pi_{2_{j}}$ is an array, the constraint $\phi_{2_{j}}$ qualifies $\pi_{2_{j}}$ 's index variables. Each $\pi_{3_{k}}$ represents one possible (scalar) value of $v_{3}$. Since we want to access the element 
Assign

$\Sigma \vdash v_{1}: \mathfrak{L}_{v_{1}}, v_{2}: \mathfrak{L}_{v_{2}}$
$\frac{\Gamma^{\prime}=\Gamma\left[\mathfrak{L}_{v_{1}} \leftarrow \Gamma\left(\mathfrak{L}_{v_{2}}\right)\right]}{\Sigma, \Gamma \vdash v_{1}=v_{2}: \Gamma^{\prime}}$

\section{Array Load}

$\Sigma \vdash v_{1}: \mathfrak{L}_{v_{1}}, v_{2}: \mathfrak{L}_{v_{2}}, v_{3}: \mathfrak{L}_{v_{3}}$

$\Gamma \vdash{ }_{j} \mathfrak{L}_{v_{2}}:\left\langle\pi_{2_{j}}, \phi_{2_{j}}\right\rangle\left(i\right.$ index of $\left.\pi_{2_{j}}\right)$

$\Gamma \vdash_{k} \mathfrak{L}_{v_{3}}:\left\langle * \pi_{3_{k}}, \phi_{3_{k}}\right\rangle$

$\Gamma \vdash_{l} \pi_{2_{j}}:\left\langle\pi_{t_{j l}}, \phi_{t_{j l}}\right\rangle$

$\phi_{2 j k}^{\prime}=U\left(\phi_{2_{j}}\left[i^{\prime}-\pi_{3_{k}} / i^{\prime}\right]\right)$

$\phi_{t_{j k l}}^{\prime \prime}=\phi_{t_{j l}} \downarrow_{\mathfrak{s}\left(\pi_{2}\right)} \phi_{2_{j k}}^{\prime}$

$\frac{\Gamma^{\prime}=\Gamma\left[\mathfrak{L}_{v_{1}} \leftarrow\left(\bigcup_{j k l}\left\langle\pi_{t_{j l}}, \phi_{t_{j k l}}^{\prime} \wedge \phi_{3_{k}}\right\rangle\right)\right]}{\Sigma, \Gamma \vdash v_{1}=v_{2}\left[v_{3}\right]: \Gamma^{\prime}}$

\section{Pointer Arithmetic}

$$
\begin{aligned}
& \Sigma \vdash v_{1}: \mathfrak{L}_{v_{1}}, v_{2}: \mathfrak{L}_{v_{2}}, v_{3}: \mathfrak{L}_{v_{3}} \\
& \Gamma \vdash_{j} \mathfrak{L}_{v_{2}}:\left\langle\pi_{2_{j}}, \phi_{2_{j}}\right\rangle \\
& \Gamma \vdash_{k} \mathfrak{L}_{v_{3}}:\left\langle * \pi_{3_{k}}, \phi_{3_{k}}\right\rangle \\
& \phi_{2}^{\prime}{ }_{j k}=\phi_{2_{j}}\left[\left(i^{\prime}-\pi_{3_{k}}\right) / i^{\prime}\right] \quad\left(i \text { index of } \pi_{2_{j}}\right) \\
& \Gamma^{\prime}=\Gamma\left[\mathfrak{L}_{v_{1}} \leftarrow\left(\bigcup_{j k}\left\langle\pi_{2_{j}}, \phi_{2_{j k}}^{\prime} \wedge \phi_{3_{k}}\right\rangle\right)\right] \\
& \Sigma, \Gamma \vdash v_{1}=v_{2} \oplus v_{3}: \Gamma^{\prime}
\end{aligned}
$$

\section{If Statement}

$$
\begin{aligned}
& \Sigma \vdash v: \mathfrak{L}_{v} \\
& \Gamma \vdash \mathfrak{L}_{v}:\left\{\left\langle * 1, \phi_{\text {true }}\right\rangle,\left\langle * 0, \phi_{\text {false }}\right\rangle\right\} \\
& \Sigma, \Gamma \vdash S_{1}: \Gamma^{\prime} \\
& \Sigma, \Gamma \vdash S_{2}: \Gamma^{\prime \prime} \\
& \Gamma_{T}=\Gamma^{\prime} \wedge \phi_{\text {true }} \\
& \Gamma_{F}=\Gamma^{\prime \prime} \wedge \phi_{\text {false }}
\end{aligned}
$$

Alloc

$$
\begin{aligned}
& \Sigma \vdash v_{1}: \mathfrak{L}_{v_{1}} \\
& \frac{\Gamma^{\prime}=\Gamma\left[\mathfrak{L}_{v_{1}} \leftarrow\left\langle\text { alloc }_{i d}\right\rangle_{i}\right] \wedge i^{\prime}=0 \quad(i \text { fresh })}{\Sigma, \Gamma \vdash v_{1}=\operatorname{alloc}\left(v_{2}\right): \Gamma^{\prime}}
\end{aligned}
$$

\section{Array Store (Fluid Update)}

$$
\begin{aligned}
& \Sigma \vdash v_{1}: \mathfrak{L}_{v_{1}}, v_{2}: \mathfrak{L}_{v_{2}}, v_{3}: \mathfrak{L}_{v_{3}} \\
& \Gamma \vdash_{j} \mathfrak{L}_{v_{1}}:\left\langle\pi_{1_{j}}, \phi_{1_{j}}\right\rangle \\
& \Gamma \vdash \mathfrak{L}_{v_{2}}:\left\{\left\langle\pi_{2_{1}}, \phi_{2_{1}}\right\rangle \ldots\left\langle\pi_{2_{n}}, \phi_{2_{n}}\right\rangle\right\}\left(i_{k} \text { index of } \pi_{2_{k}}\right) \\
& \Gamma \vdash_{l} \mathfrak{L}_{v_{3}}:\left\langle * \pi_{3_{l}}, \phi_{3_{l}}\right\rangle \\
& \Gamma^{\prime}=\left\{\begin{aligned}
\pi \leftarrow \Gamma(\pi) \text { if } \pi \notin\left\{\pi_{2_{1}}, \ldots \pi_{2_{n}}\right\} \\
\pi \leftarrow\left\{\left\langle\pi_{k}^{\prime}, \phi_{k}^{\prime} \wedge \neg \bigvee_{k l}\left(U\left(\phi_{2_{k}}\left[i_{k}^{\prime}-\pi_{3_{l}} / i_{k}^{\prime}\right]\right) \wedge \phi_{3_{l}}\right)\right\rangle\right. \\
\left.\quad \mid\left\langle\pi_{k}^{\prime}, \phi_{k}^{\prime}\right\rangle \in \Gamma\left(\pi_{2_{k}}\right)\right\} \text { if } \pi=\pi_{2_{k}} \in\left\{\pi_{2_{1}}, \ldots \pi_{2_{n}}\right\}
\end{aligned}\right. \\
& \Gamma^{\prime \prime}=\left\{\begin{array}{l}
\pi_{2_{1}} \leftarrow\left(\bigcup_{j l}\left\langle\pi_{1_{j}}, U\left(\phi_{2_{1}}\left[i_{1}^{\prime}-\pi_{3_{l}} / i_{1}^{\prime}\right]\right) \wedge \phi_{3_{l}} \wedge \phi_{1_{j}}\right\rangle\right) \\
\cdots \\
\pi_{2_{n}} \leftarrow\left(\bigcup_{j l}\left\langle\pi_{1_{j}}, U\left(\phi_{2_{n}}\left[i_{n}^{\prime}-\pi_{3_{l}} / i_{n}^{\prime}\right]\right) \wedge \phi_{3_{l}} \wedge \phi_{1_{j}}\right\rangle\right)
\end{array}\right.
\end{aligned}
$$

\section{Predop}

$$
\begin{aligned}
& \Sigma \vdash v_{1}: \mathfrak{L}_{v_{1}}, v_{2}: \mathfrak{L}_{v_{2}}, v_{3}: \mathfrak{L}_{v_{3}} \\
& \phi_{j k}=\left(\overline{\pi_{2 j}} \text { predop } \overline{\pi_{3_{k}}}\right) \wedge \phi_{2_{j}} \wedge \phi_{3_{k}} \\
& \phi_{j k}^{\text {true }}=\operatorname{Eliminate}\left(\exists \boldsymbol{f}_{\mathbf{2}}, \boldsymbol{f}_{\mathbf{3}} \cdot \phi_{j k}\right) \\
& \frac{\Gamma^{\prime}=\Gamma\left[\mathfrak{L}_{v_{1}} \leftarrow\left(\bigcup_{j k}\left\langle * 1, \phi_{j k}^{\text {true }}\right\rangle \cup\left\langle * 0, \neg \phi_{j k}^{\text {true }}\right\rangle\right)\right]}{\Sigma, \Gamma \vdash v_{1}=v_{2} \text { predop } v_{3}: \Gamma^{\prime}} \\
& \Gamma_{P}=\text { Parametrize }(\Gamma) \\
& \Sigma \vdash v: \mathfrak{L}_{v} \\
& \Gamma_{P} \vdash \mathfrak{L}_{v}:\left\{\left\langle * 1, \phi_{\text {true }}\right\rangle,\left\langle * 0, \phi_{\text {false }}\right\rangle\right\} \\
& \Sigma, \Gamma_{P} \vdash S: \Gamma^{\prime \prime} \Gamma^{\prime \prime \prime}=\Gamma^{\prime \prime} \wedge \phi_{\text {true }} \\
& \Delta=\Gamma^{\prime \prime \prime}-\Gamma_{P} \quad \Delta_{n}=f i x(\Delta) \\
& \Delta_{\text {gen }}=\operatorname{Generalize}\left(\Delta_{n}\right) \\
& \frac{\Gamma_{\text {final }}=\Gamma \circ \Delta_{\text {gen }}(\text { Generalized Fluid Update })}{\Sigma, \Gamma \vdash \text { while } v \neq 0 \text { do } S \text { end }: \Gamma_{\text {final }}}
\end{aligned}
$$

\section{While Loop}$$
\Gamma \vdash_{j} \mathfrak{L}_{v_{2}}:\left\langle * \pi_{2_{j}}, \phi_{2_{k}}\right\rangle \text { (rename all index variables to fresh } \boldsymbol{f}_{\mathbf{2}} \text { ) }
$$$$
\Gamma \vdash_{k} \mathfrak{L}_{v_{3}}:\left\langle * \pi_{3_{k}}, \phi_{3_{k}}\right\rangle \text { (rename all index variables to fresh } \boldsymbol{f}_{\boldsymbol{3}} \text { ) }
$$

Fig. 2. Rules describing the basic analysis

at offset $v_{3}$ of $v_{2}$ 's target, we select the element at offset $v_{3}$ by substituting $i^{\prime}$ with $i^{\prime}-\pi_{3_{k}}$ in the constraint $\phi_{2_{j}}$, which effectively increments the value of $i^{\prime}$ by $\pi_{3_{k}}$. Now, we need to determine the targets of those elements of $\pi_{2_{j}}$ whose indices are consistent with $\phi_{2_{j k}}^{\prime}$; hence, we compute $\phi_{t_{j l}} \downarrow_{\mathfrak{I}\left(\pi_{2_{j}}\right)} \phi_{2_{j k}}^{\prime}$ (recall Section (3) for each target $\pi_{t_{j l}}$ of $\pi_{2_{j}}$. The following example illustrates this rule.

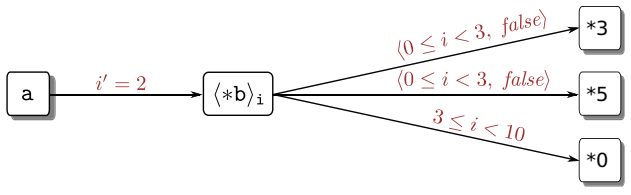

Fig. 3. Here, a points to the third element of an array of size 10, whose first three elements have the value 3 or 5 , and the remaining elements are 0
Example 2. Consider performing $\mathrm{t}=\mathrm{a}[1]$ on the symbolic heap abstraction shown in Figure 3 Here, $\mathfrak{L}_{v_{2}}$ is the memory location labeled a, the only target $\pi_{2_{j}}$ of $\mathfrak{L}_{v_{2}}$ is $\langle * \mathrm{~b}\rangle_{i}$, and the only $\pi_{3_{k}}$ is 1 . The constraint $\phi_{2_{j k}}^{\prime}$ is $U\left(\left(i^{\prime}=2\right)\left[i^{\prime} / i^{\prime}-1\right]\right)$, which is $i=3$. Thus, we need to determine the target(s) of the fourth 
element in array $\langle * \mathrm{~b}\rangle_{i}$. There are three targets $\pi_{t_{j l}}$ of $\langle * \mathrm{~b}\rangle_{i}: * 3, * 5, * 0$; hence, we compute $\phi_{t_{j k l}}^{\prime}$ once for each $\pi_{t_{j k l}}$. The only satisfiable edge under constraint $i=3$ is the edge to $* 0$ and we compute Eliminate $(\exists i .3 \leq i<10 \wedge i=3)$, which is true. Thus, the value of $t$ is guaranteed to be 0 after this statement.

The Array Store rule performs a fluid update on an abstract memory location associated with an array. In this rule, each $\pi_{2_{k}} \in\left\{\pi_{2_{1}} \ldots \pi_{2_{n}}\right\}$ represents an array location, a subset of whose elements may be written to as a result of this store. $\Gamma^{\prime}$ represents the symbolic heap abstraction after removing the points-to edges from array elements that are written to by this store while preserving all other edges, and $\Gamma^{\prime \prime}$ represents all edges added by this store. Hence, $\Gamma^{\prime}$ and $\Gamma^{\prime \prime}$ are unioned to obtain the symbolic heap abstraction after the store. Note that $\Gamma^{\prime}$ preserves the existing targets of any access path $\pi \notin\left\{\pi_{2_{1}} \ldots \pi_{2_{n}}\right\}$. The points-to targets of those elements of $\pi_{2_{1}}, \ldots \pi_{2_{n}}$ that are not affected by this store are also preserved in $\Gamma^{\prime}$ while elements that are written to by the store are killed in $\Gamma^{\prime}$. This is because elements that are updated by the store must satisfy $U\left(\phi_{2_{k}}\left[i_{k}^{\prime}-\pi_{3_{l}} / i_{k}^{\prime}\right]\right) \wedge \phi_{3_{l}}$ for some $k, l$ such that the edge to $\pi_{k}^{\prime}$ is effectively killed for those elements updated by the store. On the other hand, elements that are not affected by the store are guaranteed not to satisfy $U\left(\phi_{2_{k}}\left[i_{k}^{\prime}-\pi_{3_{l}} / i_{k}^{\prime}\right]\right) \wedge \phi_{3_{l}}$ for any $k$, l, i.e., $\neg \bigvee_{k l}\left(U\left(\phi_{2_{k}}\left[i_{k}^{\prime}-\pi_{3_{l}} / i_{k}^{\prime}\right]\right) \wedge \phi_{3_{l}}\right)=$ false, and the existing edge to $\pi_{k}^{\prime}$ is therefore preserved. Note that negation is only used in the Fluid Update rule; the soundness of negation, and therefore the correctness of fluid updates, relies on using bracketing constraints.

Example 3. Consider the effect of the following store instructions

$$
\mathrm{a}[\mathrm{k}]=7 ; \mathrm{a}[\mathrm{m}]=3 ;
$$

on Figure 3. Suppose $\mathrm{k}$ and $\mathrm{m}$ are symbolic, i.e., their values are unknown. When processing the statement $\mathrm{a}[\mathrm{k}]=7$, the only location stored into, i.e., $\pi_{2_{k}}$, is $\langle * \mathrm{~b}\rangle_{i}$. The only $\pi_{3_{l}}$ is $\mathrm{k}$ under true, and the only $\pi_{1_{j}}$ is $* 7$ under true. The elements of $\langle * \mathrm{~b}\rangle_{\mathrm{i}}$ updated by the store are determined from $U\left(\left(i^{\prime}=2\right)\left[i^{\prime}-\mathrm{k} / i^{\prime}\right]\right)=(i=\mathrm{k}+2)$. Thus, a new edge is added from $\langle * \mathrm{~b}\rangle_{\mathrm{i}}$ to $* 7$ under $i=\mathrm{k}+2$ but all outgoing edges from $\langle * \mathrm{~b}\rangle_{\mathrm{i}}$ are preserved under the constraint $i \neq \mathrm{k}+2$. Thus, after this statement, the edge from $\langle * \mathrm{~b}\rangle_{\mathrm{i}}$ to $* 3$ and $* 5$ are qualified by the constraint $\langle 0 \leq$ $i<3 \wedge i \neq k+2$, false $\rangle$, and the edge to $* 0$ is qualified by $3 \leq i<10 \wedge i \neq k+2$. The instruction $\mathrm{a}[\mathrm{m}]=3$ is processed similarly; Figure 4 shows the resulting

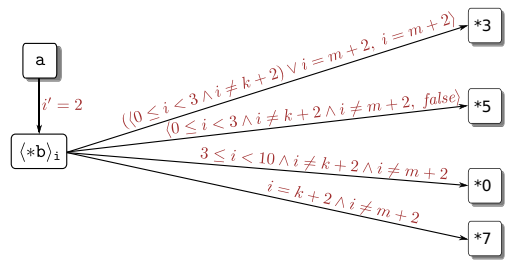

Fig. 4. Graph after processing the statements in Example 3

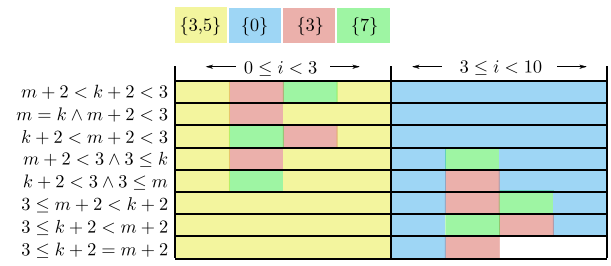

Fig. 5. Colored rectangles illustrates the partitions in Example 3 , equations on the left describe the ordering between variables 
symbolic heap abstraction after these store instructions. Note that if $k=m$, the graph correctly reflects $\mathrm{a}[\mathrm{k}]$ must be 3 . This is because if $k=m$, the constraint on the edge from $\langle * \mathrm{~b}\rangle_{\mathrm{i}}$ to $* 7$ is unsatisfiable. Since the only other feasible edge under the constraint $i=k+2$ is the one to $* 3, k=m$ implies a[k] must be 3 .

As Example 3 illustrates, fluid updates do not construct explicit partitions of the heap when different symbolic values are used to store into an array. Instead, all "partitions" are implicitly encoded in the constraints, and while the constraint solver may eventually need to analyze all of the cases, in many cases it will not because a query is more easily shown satisfiable or unsatisfiable for other reasons. As a comparison, in Example 3. approaches that eagerly construct explicit partitions may be forced to enumerate all partitions created due to stores using symbolic indices. Figure 5 shows that eight different heap configurations arise after performing the updates in Example 3. In fact, only one more store using a symbolic index could create over 50 different heap configurations.

In the Pointer Arithmetic rule, the index variable $i^{\prime}$ is replaced by $i^{\prime}-\pi_{3_{k}}$ in the index constraint $\phi_{2_{j}}$, effectively incrementing the value of $i^{\prime}$ by $v_{3}$. We also discuss the Predop rule, since some complications arise when array elements are used in predicates. In this rule, we make use of an operation $\bar{\pi}$ which converts an access path to a term in the constraint language:

$$
\begin{aligned}
\overline{\pi_{R}} & =\pi_{R} \quad \text { if } \pi_{R} \in\left\{c, \mathfrak{L}_{v}, \text { alloc }_{i d}\right\} & \overline{* \pi} & =\operatorname{deref}(\bar{\pi}) \\
\overline{\langle\pi\rangle_{i}} & =\operatorname{select}(\bar{\pi}, i) & \overline{\pi_{1} \text { intop } \pi_{2}} & =\overline{\pi_{1}} \text { intop } \overline{\pi_{2}}
\end{aligned}
$$

In this rule, notice that index variables used in the targets of $\mathfrak{L}_{v_{2}}$ and $\mathfrak{L}_{v_{3}}$ are first renamed to fresh variables $\boldsymbol{f}_{\mathbf{2}}$ and $\boldsymbol{f}_{\mathbf{3}}$ to avoid naming conflicts and are then existentially quantified and eliminated similar to computing $\phi_{1} \downarrow_{I} \phi_{2}$. The renaming of index variables is necessary since naming conflicts arise when $\left\langle * \pi_{2_{j}}, \phi_{2_{j}}\right\rangle$ and $\left\langle * \pi_{3_{k}}, \phi_{3_{k}}\right\rangle$ refer to different elements of the same array 2

In the If Statement rule, observe that the constraint under which $v \neq 0$ evaluates to true (resp. false) is conjoined with all the edge constraints in $\Gamma^{\prime}$ (resp. $\left.\Gamma^{\prime \prime}\right)$; hence, the analysis is path-sensitive. We defer discussion of the While Loop rule until Section 5 .

\subsection{Soundness of the Memory Abstraction}

We now state the soundness theorem for our memory abstraction. For a concrete store $S$, we use the notation $S\left(l_{s}, l_{t}\right)=$ true if $S\left(l_{s}\right)=l_{t}$ and $S\left(l_{s}, l_{t}\right)=$ false otherwise. Similarly, we write $\Gamma\left(\pi_{s}, \pi_{t}\right)=\phi$ to denote that the bracketing constraint associated with the edge from $\pi_{s}$ to $\pi_{t}$ is $\phi$, and $\phi$ is false if there is no edge between $\pi_{s}$ and $\pi_{t}$. Recall that $\mathfrak{I}(\pi)$ denotes the set of index variables in $\pi$, and we write $\sigma_{\Im(\pi)}$ to denote some concrete assignment to the index variables

\footnotetext{
${ }^{2}$ Quantifier elimination performed here may not be exact; but since we use bracketing constraints, we compute quantifier-free over- and underapproximations. For instance, 11] presents a technique for computing covers of existentially quantified formulas in combined theories involving uninterpreted functions. Another alternative is to allow quantification in our constraint language.
} 
in $\mathfrak{I}(\pi) ; \sigma_{\Im(\pi)}^{\prime}$ is an assignment to $\mathfrak{I}(\pi)$ with all index variables primed. The notation $\sigma(\phi)$ applies substitution $\sigma$ to $\phi$. Finally, we use a function $\operatorname{eval}^{\star}(\phi, E, S)$ for $\star \in\{+,-\}$ which evaluates the truth value of the necessary and sufficient conditions of constraint $\phi$ for some concrete environment $E$ and concrete store $S$; this function is precisely defined in [30].

Definition 2 (Agreement). We say a concrete environment and concrete store $(E, S)$ agrees with abstract environment and abstract store $(\Sigma, \Gamma)$ (written $(E, S) \sim(\Sigma, \Gamma))$ if and only if the following conditions hold:

1. $E$ and $\Sigma$ have the same domain

2. If $S\left(l_{s}, l_{t}\right)=b$ and $\Gamma\left(\pi_{s}, \pi_{t}\right)=\left\langle\phi^{+}, \phi^{-}\right\rangle$, then for all substitutions $\sigma_{\Im}\left(\pi_{s}\right)$, $\sigma_{\Im\left(\pi_{t}\right)}^{\prime}$ such that $l_{s} \in \gamma_{c}\left(E, S, \pi_{s}, \sigma_{\Im\left(\pi_{s}\right)}\right)$ and $l_{t} \in \gamma_{c}\left(E, S, \pi_{t}, \sigma_{\Im\left(\pi_{t}\right)}^{\prime}\right)$, we have:

$$
\operatorname{eval}^{-}\left(\sigma^{\prime}\left(\sigma\left(\phi^{-}\right)\right), E, S\right) \Rightarrow b \Rightarrow \operatorname{eval}^{+}\left(\sigma^{\prime}\left(\sigma\left(\phi^{+}\right)\right), E, S\right)
$$

Theorem 1 (Soundness). Let $P$ be any program. If $(E, S) \sim(\Sigma, \Gamma)$, then

$$
E, S \vdash P: S^{\prime} \Rightarrow\left(\Sigma, \Gamma \vdash P: \Gamma^{\prime} \wedge\left(E, S^{\prime}\right) \sim\left(\Sigma, \Gamma^{\prime}\right)\right)
$$

We sketch the proof of Theorem 1 in the extended version [30].

\section{$5 \quad$ Fluid Updates in Loops}

In loop-free code, a store modifies one array element, but stores inside a loop often update many elements. In this section, we describe a technique to overand underapproximate the set of concrete elements updated in loops. The main idea of our approach is to analyze the loop body and perform a fixed-point computation parametric over an iteration counter. Once a fixed-point is reached, we use quantifier elimination to infer elements that may and must be modified by the loop 3

\subsection{Parametrizing the Symbolic Heap Abstraction}

When analyzing loops, our analysis first identifies the set of scalars modified by the loop; we call such values loop-dependent scalars. We then infer equalities relating each loop-dependent scalar to the unique iteration counter $k$ for that loop. The iteration counter $k$ is assumed to be initialized to 0 at loop entry and is incremented by one along the back edge of the loop. We say that a loopdependent value $i$ is linear with respect to the loop if $i-i_{0}=c * k$ for some constant $c \neq 0$. We compute a set of equalities relating loop-dependent scalars to the iteration counter using standard linear invariant generation techniques [12, 13]. At loop entry, we use these linear equalities to modify $\Gamma$ as follows:

\footnotetext{
${ }^{3}$ In this section, we assume no pointer arithmetic occurs in loops; our implementation, however, does not make this restriction.
} 
- Let $\pi$ be a linear loop-dependent scalar with the linear relation $\pi=\pi_{0}+c * k$, and let $\left\langle * \pi_{t}, c_{t}\right\rangle \in \Gamma(\pi)$. Then, replace $\pi_{t}$ by $\pi_{t}+c * k$.

- Let $\pi$ be a loop-dependent value not linear in $k$. Then, $\Gamma(\pi) \leftarrow\{\langle\top$, true $\rangle$.

Thus, all loop-dependent scalars are expressed in terms of their value at iteration $k$ or $T$; analysis of the loop body proceeds as described in Section 4

Example 4. Consider the send_packets function from Section 1 Here, we infer the equality $j=j_{0}+2 k$, and $\Gamma$ initially contains an edge from $j$ to $*\left(j_{0}+2 k\right)$.

\subsection{Fixed-Point Computation}

Next, we perform a fixed-point computation (parametric on $k$ ) over the loop's net effect on the symbolic heap abstraction. This is necessary because there may be loop carried dependencies through heap reads and writes. We define the net effect of the loop on the symbolic heap abstraction during some iteration $k$ as the effect set:

Definition 3. (Effect Set $\boldsymbol{\Delta}$ ) Let $\Gamma^{\prime}$ be a symbolic heap obtained by performing fluid updates on $\Gamma$. Let $\Delta=\Gamma^{\prime}-\Gamma$ be the set of edges such that if $\phi$ qualifies edge $e$ in $\Gamma$ and $\phi^{\prime}$ qualifies $e$ in $\Gamma^{\prime}$, then $\Delta$ includes $e$ under constraint $\phi^{\prime} \wedge \neg \phi$ (where $\phi=$ false if $e \notin \Gamma$ ). We call $\Delta$ the effect set of $\Gamma^{\prime}$ with respect to $\Gamma$.

Example 5. Figure 6] shows the effect set of the loop in send_packets after analyzing its body once. (Edges with false constraints are not shown.) Note that the constraints qualifying edges in this figure are parametric over $k$.

We define $\Gamma \circ \Delta$ as the generalized fluid update that applies $\Delta$ to $\Gamma$ :

Definition 4. $(\boldsymbol{\Gamma} \circ \boldsymbol{\Delta})$ Let $\pi$ be a location in $\Gamma$ and let $S_{\pi}$ denote the edges in $\Delta$ whose source is $\pi$. Let $\delta\left(S_{\pi}\right)$ be the disjunction of constraints qualifying edges in $S_{\pi}$, and let $I$ be the set of index variables used in the target locations in $S_{\pi}$ but not the source. Let $\operatorname{Update}(\pi)=\operatorname{Eliminate}\left(\exists I . \delta\left(S_{\pi}\right)\right)$. Then, for each $\pi \in \Gamma$ :

$$
(\Gamma \circ \Delta)[\pi]=(\Gamma(\pi) \wedge \neg \operatorname{Update}(\pi)) \cup S_{\pi}
$$

The above definition is a straightforward generalization of the fluid update operation given in the Store rule of Figure 2. Instead of processing a single store, it reflects the overall effect on $\Gamma$ of a set of updates defined by $\Delta$. The fixedpoint computation is performed on $\Delta$. We denote an edge from location $\pi_{s}$ to $\pi_{t}$ qualified by constraint $\phi$ as $\left\langle\pi_{s}, \pi_{t}\right\rangle \backslash \phi$. Since we compute a least fixed point, $\left\langle\pi_{s}, \pi_{t}\right\rangle \backslash\langle$ false, true $\rangle \in \perp$ for all legal combinations (i.e., obeying type restrictions)

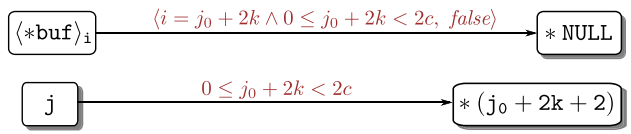

Fig. 6. The effect set after analyzing the loop body once in function send_packets 
of all $\left\langle\pi_{s}, \pi_{t}\right\rangle$ pairs. Note that the edge constraints in $\perp$ are the inconsistent bounds $\langle$ false, true $\rangle$ representing the strongest over- and underapproximations. We define a $\sqcup$ and $\sqsubseteq$ on effect sets as follows:

$$
\begin{array}{cc}
\left\langle\pi_{s}, \pi_{t}\right\rangle \backslash\left\langle\left(\phi_{n c 1} \vee \phi_{n c 2}\right),\left(\phi_{s c 1} \wedge \phi_{s c 2}\right)\right\rangle \in \Delta_{1} \sqcup \Delta_{2} & \Delta_{1} \sqsubseteq \Delta_{2} \\
\Longleftrightarrow & \Longleftrightarrow \\
\left(\left\langle\pi_{s}, \pi_{t}\right\rangle \backslash\left\langle\phi_{n c 1}, \phi_{s c 1}\right\rangle \in \Delta_{1} \wedge\right. & \left(\left(\phi_{n c 1} \Rightarrow \phi_{n c 2} \wedge \phi_{s c 2} \Rightarrow \phi_{s c 1}\right)\right. \\
\left.\left\langle\pi_{s}, \pi_{t}\right\rangle \backslash\left\langle\phi_{n c 2}, \phi_{s c 2}\right\rangle \in \Delta_{2}\right) & \forall\left\langle\pi_{s}, \pi_{t}\right\rangle \backslash\left\langle\phi_{n c 1}, \phi_{s c 1}\right\rangle \in \Delta_{1} \wedge \\
& \left.\forall\left\langle\pi_{s}, \pi_{t}\right\rangle \backslash\left\langle\phi_{n c 2}, \phi_{s c 2}\right\rangle \in \Delta_{2}\right)
\end{array}
$$

Let $\Gamma_{0}$ be the initial symbolic heap abstraction before the loop. We compute $\Gamma_{\text {entry }}^{n}$ representing the symbolic heap on entry to the $n$ 'th iteration of the loop:

$$
\Gamma_{\text {entry }}^{n}=\left\{\begin{array}{lll}
\Gamma_{0} & \text { if } & n=1 \\
\Gamma_{0} \circ\left(\Delta_{n-1}[k-1 / k]\right) & \text { if } & n>1
\end{array}\right.
$$

$\Gamma_{\text {exit }}^{n}$ is obtained by analyzing the body of the loop using $\Gamma_{\text {entry }}^{n}$ at the entry point of the loop. In the definition of $\Gamma_{\text {entry }}^{n}$, the substitution $[k-1 / k]$ normalizes the effect set with respect to the iteration counter so that values of loop-dependent scalars always remain in terms of their value at iteration $k$. We define $\Delta_{n}$ representing the total effect of the loop in $n$ iterations as follows:

$$
\Delta_{n}= \begin{cases}\perp & \text { if } n=0 \\ \left(\Gamma_{\text {exit }}^{n}-\Gamma_{\text {entry }}^{n}\right) \sqcup \Delta_{n-1} & \text { if } n>0\end{cases}
$$

First, observe that $\Delta_{n-1} \sqsubseteq \Delta_{n}$ by construction (monotonicity). Second, observe the analysis cannot create an infinite number of abstract locations because (i) arrays are represented as indexed locations, (ii) pointers can be dereferenced only as many times as their types permit, (iii) all allocations are named by their allocation site, and (iv) scalars are represented in terms of their linear relation to $k$. However, our constraint domain does not have finite ascending chains, hence, we define a widening operator on bracketing constraints (although widening was never required in our experiments). Let $\boldsymbol{\beta}$ denote the unshared literals between any constraint $\phi_{1}$ and $\phi_{2}$. Then, we widen bracketing constraints as follows:

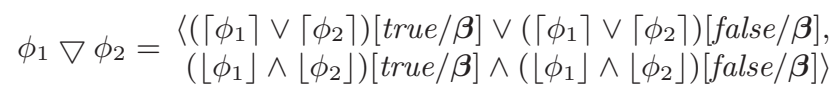

Example 6. The effect set obtained in Example 5 does not change in the second iteration; therefore the fixed-point computation terminates after two iterations.

\subsection{Generalization}

In this section, we describe how to generalize the final effect set after a fixed-point is reached. This last step allows the analysis to extrapolate from the elements modified in the $k^{\prime}$ th iteration to the set of elements modified across all iterations and is based on existential quantifier elimination.

Definition 5. (Generalizable Location) We say a location identified by $\pi$ is generalizable in a loop if (i) $\pi$ is an array, (ii) if $\pi_{i}$ is used as an index in a store to $\pi$, then $\pi_{i}$ must be a linear function of the iteration counter, and (iii) if two distinct indices $\pi_{i}$ and $\pi_{j}$ may be used to store into $\pi$, then either only $\pi_{i}$, or only $\pi_{j}$ (or neither) is used to index $\pi$ across all iterations. 
Intuitively, if a location $\pi$ is generalizable, then all writes to $\pi$ at different iterations of the loop must refer to distinct concrete elements. Clearly, if $\pi$ is not an array, different iterations of the loop cannot refer to distinct concrete elements. If an index used to store into $\pi$ is not a linear function of $k$, then the loop may update the same concrete element in different iterations. Furthermore, if two values that do not have the same relation with respect to $k$ are used to store into $\pi$, then they may update the same element in different iterations.

In order to generalize the effect set, we make use of a variable $N$ unique for each loop that represents the number of times the loop body executes. If the value of $N$ can be determined precisely, we use this exact value instead of introducing $N$. For instance, if a loop increments $i$ by 1 until $i \geq$ size, then it is easy to determine that $N=$ size $-i_{0}$, assuming the loop executes at least once 4 Finally, we generalize the effect set as follows:

- If an edge qualified by $\phi$ has a generalizable source whose target does not mention $k$, the generalized constraint is $\phi^{\prime}=\operatorname{Eliminate}(\exists k .(\phi \wedge 0 \leq k<N))$.

- If an edge qualified by $\phi$ does not have a generalizable source, the generalized constraint is $\phi^{\prime}=$ Eliminate $\langle\exists k . \phi \wedge 0 \leq k<N, \quad \forall k .0 \leq k<N \Rightarrow \phi\rangle \sqrt{5}$.

- If $\pi$ is a loop-dependent scalar, then $\Delta[\pi] \leftarrow \Delta[\pi][N / k]$.

We now briefly explain these generalization rules. If the source of an edge is generalizable, for each iteration of the loop, there exists a corresponding concrete element of the array that is updated during this iteration; thus, $k$ is existentially quantified in both the over- and underapproximation. The constraint after the existential quantifier elimination specifies the set of concrete elements updated by the loop. If the source is not generalizable, it is unsafe to existentially quantify $k$ in the underapproximation since the same concrete element may be overwritten in future iterations. One way to obtain an underapproximation is to universally quantify $k$ because if the update happens in all iterations, then the update must happen after the loop terminates. According to the last rule, loop-dependent scalar values are assigned to their value on termination. Once the effect set is generalized, we apply it to $\Gamma_{0}$ to obtain the final symbolic heap abstraction after the loop.

Example 7. Consider the effect set given in Figure 6. In the send_packets function, $\langle * \text { buf }\rangle_{i}$ is generalizable since $\mathrm{j}$ is linear in $k$ and no other value is used to index $\langle * \text { buf }\rangle_{i}$. Furthermore, if the loop executes, it executes exactly $c$ times; thus $N=c$. To generalize the edge from $\langle * \text { buf }\rangle_{i}$ to $*$ NULL, we perform quantifier elimination on $\left\langle\exists k . i=j_{0}+2 k \wedge 0 \leq j_{0}+2 k<2 c \wedge 0 \leq k<c\right.$, false $\rangle$, which yields $\left\langle j_{0} \leq i \wedge i<j_{0}+2 c \wedge\left(i-j_{0}\right) \% 2=0\right.$, false $\rangle$. Since $j_{0}$ is 0 at loop entry, after applying the generalized effect set to $\Gamma_{0}$, we obtain the graph from Figure 1 .

\footnotetext{
${ }^{4}$ Even though it is often not possible to determine the exact value of $N$, our analysis utilizes the constraint $\left(\forall k .0 \leq k<N \Rightarrow \neg \phi_{\text {term }}(k)\right) \wedge \phi_{\text {term }}(N)$ stating that the termination condition $\phi_{\text {term }}$ does not hold on iterations before $N$ but holds at the $N$ 'th iteration. Our analysis takes this "background axiom" into account when determining satisfiability and validity.

${ }^{5}$ We can eliminate a universally quantified variable $k$ from $\forall k . \phi$ by eliminating existentially quantified $k$ in the formula $\neg \exists k . \neg \phi$.
} 


\section{Implementation and Extensions}

We have implemented the ideas presented in this paper in the Compass program verification framework for analyzing $\mathrm{C}$ programs. For solving constraints, Compass utilizes a custom SMT solver called Mistral [14], which also provides support for simplifying constraints. Compass does not assume type safety and handles casts soundly using a technique based on physical subtyping [15]. Compass supports most features of the $\mathrm{C}$ language, including structs, unions, multidimensional arrays, dynamic memory allocation, and pointer arithmetic. To check buffer overruns, Compass also tracks buffer and allocation sizes. For interprocedural analysis, Compass performs a path- and context-sensitive summarybased analysis. Loop bodies are analyzed in isolation before the function or loop in which they are defined; thus techniques from Section 5 extend to nested loops.

While the language defined in Section 2 only allows loops with a single exit point, techniques described in this paper can be extended to loops with multiple break points by introducing different iteration counters for each backedge, similar to the technique used in [16] for complexity analysis.

Compass allows checking arbitrary assertions using a static_assert(...) primitive, which can be either manually or automatically inserted (e.g., for memory safety properties). The static_assert primitive also allows for checking quantified properties, such as "all elements of arrays a and b are equal" by writing:

static_assert (buffer_size(b) == buffer_size(a));

for $\left(i=0 ; i<b u f f e r \_s i z e(a)\right.$; $\left.i++\right)$ static_assert $(a[i]==b[i])$;

\section{Experiments}

\subsection{Case Study on Example Benchmarks}

To demonstrate the expressiveness of our technique, we evaluate it on 28 challenging array benchmarks available at http://www.stanford.edu/ ${ }^{\text {tdillig/array.tar.gz }}$ and shown in Figure 7. The functions init and init_noncost initialize all elements of an array to a constant and an iteration-dependent value respectively. init_partial initializes part of the array, and init_even initializes even positions. 2D_array_init initializes a 2-dimensional array using a nested loop. The programs labeled _buggy exhibit subtle bugs, such as off-by-one errors. Various versions of copy copy all, some, or odd elements of an array to another array. reverse reverses elements, while swap (shown in Figure 8) swaps the contents of two arrays. double_swap invokes swap twice and checks that both arrays are back in their initial state. strcpy, strlen, and memcpy implement the functionality of the standard C library functions and assert their correctness. find (resp. find_first_nonnull) looks for a specified (resp. non-null) element and returns its index (or -1 if element is not found). append appends the contents of one array to another, and merge_interleave interleaves odd and even-numbered elements of two arrays into a result array. The function alloc_fixed_size initializes all elements of a double array to a freshly allocated array of fixed size, and then checks that buffer 


\begin{tabular}{|l|l|r|c|l|}
\hline Program & Time & Memory & $\begin{array}{c}\text { \#Sat } \\
\text { queries }\end{array}$ & $\begin{array}{l}\text { Solve } \\
\text { time }\end{array}$ \\
\hline init & $0.01 \mathrm{~s}$ & $<1 \mathrm{MB}$ & 172 & $0 \mathrm{~s}$ \\
\hline init_nonconst & $0.02 \mathrm{~s}$ & $<1 \mathrm{MB}$ & 184 & $0.01 \mathrm{~s}$ \\
\hline init_partial & $0.01 \mathrm{~s}$ & $<1 \mathrm{MB}$ & 166 & $0.01 \mathrm{~s}$ \\
\hline init_partial_buggy & $0.02 \mathrm{~s}$ & $<1 \mathrm{MB}$ & 168 & $0 \mathrm{~s}$ \\
\hline init_even & $0.04 \mathrm{~s}$ & $<1 \mathrm{MB}$ & 146 & $0.04 \mathrm{~s}$ \\
\hline init_even_buggy & $0.04 \mathrm{~s}$ & $<1 \mathrm{MB}$ & 166 & $0.03 \mathrm{~s}$ \\
\hline 2D_array_init & $0.04 \mathrm{~s}$ & $<1 \mathrm{MB}$ & 311 & $0.04 \mathrm{~s}$ \\
\hline copy & $0.01 \mathrm{~s}$ & $<1 \mathrm{MB}$ & 209 & $0.01 \mathrm{~s}$ \\
\hline copy_partial & $0.01 \mathrm{~s}$ & $<1 \mathrm{MB}$ & 220 & $0.01 \mathrm{~s}$ \\
\hline copy_odd & $0.04 \mathrm{~s}$ & $<1 \mathrm{MB}$ & 243 & $0.02 \mathrm{~s}$ \\
\hline copy_odd_buggy & $0.05 \mathrm{~s}$ & $<1 \mathrm{MB}$ & 246 & $0.05 \mathrm{~s}$ \\
\hline reverse & $0.03 \mathrm{~s}$ & $<1 \mathrm{MB}$ & 273 & $0.01 \mathrm{~s}$ \\
\hline reverse_buggy & $0.04 \mathrm{~s}$ & $<1 \mathrm{MB}$ & 281 & $0.02 \mathrm{~s}$ \\
\hline swap & $0.12 \mathrm{~s}$ & $2 \mathrm{MB}$ & 590 & $0.11 \mathrm{~s}$ \\
\hline swap_buggy & $0.11 \mathrm{~s}$ & $2 \mathrm{MB}$ & 557 & $0.06 \mathrm{~s}$ \\
\hline double_swap & $0.16 \mathrm{~s}$ & $2 \mathrm{MB}$ & 601 & $0.1 \mathrm{~s}$ \\
\hline strcpy & $0.07 \mathrm{~s}$ & $<1 \mathrm{MB}$ & 355 & $0.04 \mathrm{~s}$ \\
\hline strlen & $0.02 \mathrm{~s}$ & $<1 \mathrm{MB}$ & 165 & $0.01 \mathrm{~s}$ \\
\hline strlen_buggy & $0.01 \mathrm{~s}$ & $<1 \mathrm{MB}$ & 89 & $0.01 \mathrm{~s}$ \\
\hline memcpy & $0.04 \mathrm{~s}$ & $<1 \mathrm{MB}$ & 225 & $0.04 \mathrm{~s}$ \\
\hline find & $0.02 \mathrm{~s}$ & $<1 \mathrm{MB}$ & 119 & $0.02 \mathrm{~s}$ \\
\hline find_first_nonnull & $0.02 \mathrm{~s}$ & $<1 \mathrm{MB}$ & 183 & $0.02 \mathrm{~s}$ \\
\hline append & $0.02 \mathrm{~s}$ & $<1 \mathrm{MB}$ & 183 & $0.01 \mathrm{~s}$ \\
\hline merge_interleave & $0.09 \mathrm{~s}$ & $<1 \mathrm{MB}$ & 296 & $0.07 \mathrm{~s}$ \\
\hline $\begin{array}{l}\text { merge_interleave } \\
\text {-buggy }\end{array}$ & $0.11 \mathrm{~s}$ & $<1 \mathrm{MB}$ & 305 & $0.09 \mathrm{~s}$ \\
\hline alloc_fixed_size & $0.02 \mathrm{~s}$ & $<1 \mathrm{MB}$ & 176 & $0.02 \mathrm{~s}$ \\
\hline alloc_fixed_size_buggy & $0.02 \mathrm{~s}$ & $<1 \mathrm{MB}$ & 172 & $0.02 \mathrm{~s}$ \\
\hline alloc_nonfixed_size & $0.03 \mathrm{~s}$ & $<1 \mathrm{MB}$ & 214 & 0.02 \\
\hline
\end{tabular}

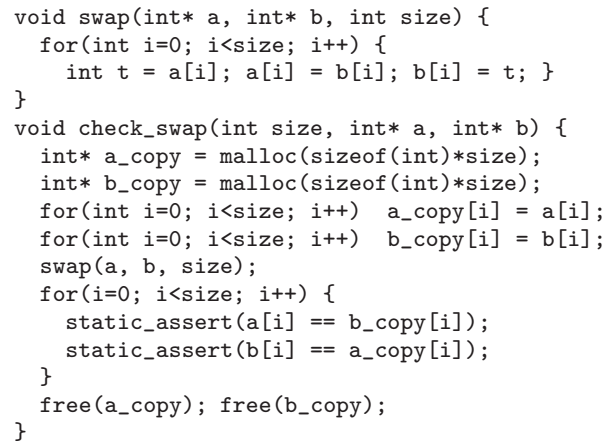

Fig. 8. Swap Function from Figure 7 The static assertions check that all elements of a and $b$ are indeed swapped after the call to the swap function. Compass verifies these

Fig. 7. Case Study assertions automatically in 0.12 seconds.

accesses to the element arrays are safe. The function alloc_nonfixed_size initializes elements of the double array a to freshly allocated arrays of different size, encoded by the elements of another array $\mathrm{b}$ and checks that accessing indices $[0, b[i-1]]$ of array $a[i]$ is safe. Compass can automatically verify the full functional correctness of all of the correct programs without any annotations and reports all errors present in buggy programs. To check functional correctness, we add static assertions as described in Section 6 and as shown in Figure 8 .

Figure 7 reports for each program the total running time, memory usage (including the constraint solver), number of queries to the SMT solver, and constraint solving time. All experiments were performed on a $2.66 \mathrm{GHz}$ Xeon workstation. We believe these experiments demonstrate that Compass reasons precisely and efficiently about array contents despite being fully automatic. As a comparison, while Compass takes 0.01 seconds to verify the full correctness of copy, the approach described in [4] reports a running time of 338.1 seconds, and the counterexample-guided abstraction refinement based approach described in [17] takes 3.65 seconds. Furthermore, our technique is naturally able to verify the correctness of programs that manipulate non-contiguous array elements (e.g., copy_odd), as well as programs that require reasoning about arrays inside other arrays (e.g., alloc_nonfixed_size). Figure 7 also shows that the analysis is memory efficient since none of the programs require more than $2 \mathrm{MB}$. We believe this to be the case because fluid updates do not create explicit partitions.

Observe that the choice of benchmarks in Figure 7 sheds light on both what our technique is good at and what it is not meant for. In particular, notice these benchmarks do not include sorting routines. While sorting is an interesting problem for invariant generation techniques, the focus of this work is improving static analysis of updates to aggregate data structures, such as arrays, through fluid 


\begin{tabular}{|l|l|l|l|l|l|}
\hline Program & Lines & Total Time & Memory & \#Sat queries & Solve Time \\
\hline hostname & 304 & $0.13 \mathrm{~s}$ & $5 \mathrm{MB}$ & 1533 & $0.12 \mathrm{~s}$ \\
\hline chroot & 371 & $0.13 \mathrm{~s}$ & $3 \mathrm{MB}$ & 1821 & $0.10 \mathrm{~s}$ \\
\hline rmdir & 483 & $1.05 \mathrm{~s}$ & $12 \mathrm{MB}$ & 3461 & $1.02 \mathrm{~s}$ \\
\hline su & 1047 & $1.86 \mathrm{~s}$ & $32 \mathrm{MB}$ & 6088 & $1.69 \mathrm{~s}$ \\
\hline $\mathbf{m v}$ & 1151 & $0.70 \mathrm{~s}$ & $21 \mathrm{MB}$ & 7427 & $0.68 \mathrm{~s}$ \\
\hline \hline Total & 3356 & $3.87 \mathrm{~s}$ & $73 \mathrm{MB}$ & 20330 & 3.61 \\
\hline
\end{tabular}

Fig. 9. Experimental results on Unix Coreutils applications

updates. As shown in Section 5 fluid updates can be combined with invariant generation techniques to analyze loops, but we do not claim that this particular invariant generation approach is the best possible. We leave as future work the combination of fluid updates and more powerful invariant generation techniques.

\subsection{Checking Memory Safety on Unix Coreutils Applications}

To evaluate the usefulness of our technique on real programs, we also check for memory safety errors on five Unix Coreutils applications 20] that manipulate arrays and pointers in complex ways. In particular, we verify the safety of buffer accesses (both buffer overruns and underruns) and pointer dereferences. However, since Compass treats integers as mathematical integers, the soundness of the buffer analysis assumes lack of integer overflow errors, which can be verified by a separate analysis. In the experiments, Compass reports zero false positives, only requiring two annotations describing inputs to main: assume(buffer_size(argv) == argc) and assume (argv! = NULL)). Compass is even able to discharge some arbitrary assertions inserted by the original programmers. Some of the buffer accesses that Compass can discharge rely on complex dependencies that are difficult even for experienced programmers to track; an interesting example is given in [30].

The chosen benchmarks are challenging for static analysis tools for multiple reasons: First, these applications heavily use arrays and string buffers, making them difficult for techniques that do not track array contents. Second, they heavily rely on path conditions and correlations between scalars used to index buffers. Finally, the behavior of these applications depends on environment choice, such as user input. Our technique is powerful enough to deal with these challenges because it is capable of reasoning about array elements, is pathsensitive, and uses bracketing constraints to capture uncertainty. To give the reader some idea about the importance of these components, $85.4 \%$ of the assertions fail if array contents are smashed and $98.2 \%$ fail if path-sensitivity is disabled.

As Figure 9 illustrates, Compass is able to analyze all applications in under 2 seconds, and the maximum memory used both for the program verification and constraint solving combined is less than $35 \mathrm{MB}$. We believe these running times and memory requirements demonstrate that the current state of Compass is useful and practical for verifying memory safety in real modest-sized $\mathrm{C}$ applications manipulating arrays, pointers, and scalars in complex ways. 


\section{Related Work}

Reasoning about unbounded data structures has a long history. Jones et al. first propose summary nodes to finitely represent lists in LISP [21], and [1] extends this work to languages with updates and introduces strong and weak updates. Representation of access paths qualified by indices is first introduced in Deutsch [22], which uses a combination of symbolic access paths and numeric abstract domains to represent may-alias pairs for recursive data structures. This technique does not address arrays, and since it does not reason about updates, negation is not a consideration. Deutsch's technique does not allow disjunctive constraints, is not path-sensitive, and does not address underapproximations.

The most basic technique for reasoning about array contents is array smashing, which represents all elements with one summary node and only allows weak updates [2]. Gopan et al. propose a 3-valued logic based framework to discover relationships about values of array elements [4]. This technique isolates individual elements to perform strong updates and places elements that share a common property into a partition (usually a contiguous range), and relevant partitions are heuristically inferred. In contrast, our approach does not need to distinguish between strong and weak updates or concretize individual elements; it can also naturally express invariants about non-contiguous array elements. Furthermore, our approach obviates the need for explicit partitioning, and effectively delays decisions about partitions until constraint solving. While many factors contribute to the overall performance of program analysis systems, we believe our tool's significantly better performance over [4] is largely due to avoiding the construction of explicit partitions. Jhala and McMillan propose a technique similar to [4] for reasoning about arrays, but their technique is based on counterexample guided abstraction refinement and interpolation [17]. This approach also only reasons about contiguous ranges and constructs explicit partitions. Furthermore, the predicates used in the abstraction belong to a finite language to guarantee convergence.

Many techniques have been proposed for generating invariants about elements of unbounded data structures [18, 19, 23 26]. Some of these techniques can reason about complex data invariants, such as sortedness, which is orthogonal to the ability to perform fluid updates. Unlike these approaches whose goal is to discover complex invariants about array elements, our goal is to design an expressive pointer and value analysis that unifies reasoning about pointers, scalars, and arrays. However, we believe these techniques can be gainfully combined.

Concepts similar to the iteration counter from Section 5 have been previously proposed. For example, Gulwani et al. [16] use an iteration counter for performing complexity analysis. The invariant generation technique described in [19] also uses a combination of an iteration counter combined with quantifier elimination.

Our technique uses bracketing constraints to represent both over- and underapproximations to naturally handle imprecision and uncertainty. Furthermore, bracketing constraints allow for a sound negation operation in the presence of approximations. The idea of over- and underapproximations has been proposed 
previously in the context of abstract interpretation by Schmidt [27]; however, the techniques presented there are not concerned with negation. In this paper, we share the goal of gracefully handling imprecision when analyzing unbounded data structures with [28], which presents a compositional shape analysis based on separation logic. In contrast to 28] which focuses exclusively on recursive pointer data structures, such as linked lists, this paper focuses on arrays. We believe our approach can be extended to at least some useful recursive data structures, such as lists, and we leave this extension as future work.

\section{References}

1. Chase, D.R., Wegman, M., Zadeck, F.K.: Analysis of pointers and structures. In: PLDI, pp. 296-310. ACM, New York (1990)

2. Blanchet, B., Cousot, P., Cousot, R., Feret, J., Mauborgne, L., Miné, A., Monniaux, D., Rival, X.: Design and implementation of a special-purpose static program analyzer for safety-critical real-time embedded software (2002)

3. Reps, T.W., Sagiv, S., Wilhelm, R.: Static program analysis via 3-valued logic. In: Alur, R., Peled, D.A. (eds.) CAV 2004. LNCS, vol. 3114, pp. 15-30. Springer, Heidelberg (2004)

4. Gopan, D., Reps, T., Sagiv, M.: A framework for numeric analysis of array operations. In: POPL, pp. 338-350. ACM, New York (2005)

5. Aiken, A., Bugrara, S., Dillig, I., Dillig, T., Hackett, B., Hawkins, P.: An overview of the saturn project. In: PASTE, pp. 43-48. ACM, New York (2007)

6. Ball, T., Rajamani, S.: The slam project: debugging system software via static analysis. In: POPL, NY, USA, pp. 1-3 (2002)

7. Lee, S., Cho, D.: Packet-scheduling algorithm based on priority of separate buffers for unicast and multicast services. Electronics Letters 39(2), 259-260 (2003)

8. Nguyen, K., Nguyen, T., Cheung, S.: P2p streaming with hierarchical network coding (July 2007)

9. Landi, W., Ryder, B.G.: A safe approximate algorithm for interprocedural aliasing. SIGPLAN Not. 27(7), 235-248 (1992)

10. Cooper, D.: Theorem proving in arithmetic without multiplication. Machine Intelligence 7, 91-100 (1972)

11. Gulwani, S., Musuvathi, M.: Cover algorithms. In: Drossopoulou, S. (ed.) ESOP 2008. LNCS, vol. 4960, pp. 193-207. Springer, Heidelberg (2008)

12. Karr, M.: Affine relationships among variables of a program. A.I., 133-151 (1976)

13. Cousot, P., Halbwachs, N.: Automatic discovery of linear restraints among variables of a program. In: POPL, pp. 84-96. ACM, New York (1978)

14. Dillig, I., Dillig, T., Aiken, A.: Cuts from proofs: A complete and practical technique for solving linear inequalities over integers. In: Bouajjani, A., Maler, O. (eds.) CAV 2009. LNCS, vol. 5643, pp. 233-247. Springer, Heidelberg (2009)

15. Chandra, S., Reps, T.: Physical type checking for c. SIGSOFT 24(5), 66-75 (1999)

16. Gulwani, S., Mehra, K., Chilimbi, T.: SPEED: precise and efficient static estimation of program computational complexity. In: POPL, pp. 127-139 (2009)

17. Jhala, R., Mcmillan, K.L.: Array abstractions from proofs. In: Damm, W., Hermanns, H. (eds.) CAV 2007. LNCS, vol. 4590, pp. 193-206. Springer, Heidelberg (2007)

18. Halbwachs, N., Péron, M.: Discovering properties about arrays in simple programs. In: PLDI, pp. 339-348. ACM, New York (2008) 
19. Kovacs, L., Voronkov, A.: Finding loop invariants for programs over arrays using a theorem prover. In: Chechik, M., Wirsing, M. (eds.) FASE 2009. LNCS, vol. 5503, pp. 470-485. Springer, Heidelberg (2009)

20. http://www.gnu.org/software/coreutils/Unix coreutils

21. Jones, N., Muchnick, S.: Flow analysis and optimization of LISP-like structures. In: POPL, pp. 244-256. ACM, New York (1979)

22. Deutsch, A.: Interprocedural may-alias analysis for pointers: Beyond k-limiting. In: PLDI, pp. 230-241. ACM, New York (1994)

23. Allamigeon, X.: Non-disjunctive numerical domain for array predicate abstraction. In: Drossopoulou, S. (ed.) ESOP 2008. LNCS, vol. 4960, pp. 163-177. Springer, Heidelberg (2008)

24. Gulwani, S., McCloskey, B., Tiwari, A.: Lifting abstract interpreters to quantified logical domains. In: POPL, pp. 235-246. ACM, New York (2008)

25. Seghir, M., Podelski, A., Wies, T.: Abstraction Refinement for Quantified Array Assertions. In: Palsberg, J., Su, Z. (eds.) SAS 2009. LNCS, vol. 5673, pp. 3-18. Springer, Heidelberg (2009)

26. Flanagan, C., Qadeer, S.: Predicate abstraction for software verification. In: POPL, pp. 191-202. ACM, New York (2002)

27. Schmidt, D.A.: A calculus of logical relations for over- and underapproximating static analyses. Sci. Comput. Program. 64(1), 29-53 (2007)

28. Calcagno, C., Distefano, D., O’Hearn, P., Yang, H.: Compositional shape analysis by means of bi-abduction. In: POPL, pp. 289-300. ACM, New York (2009)

29. Cousot, P.: Verification by abstract interpretation. In: Dershowitz, N. (ed.) Verification: Theory and Practice. LNCS, vol. 2772, pp. 243-268. Springer, Heidelberg (2004)

30. Dillig, I., Dillig, T., Aiken, A.: Fluid updates: Beyond strong vs. weak updates (extended version), http://www.stanford.edu/ isil/esop-extended.pdf 